\title{
Cavitation damage observations within scroll expander lubrication systems
}

\author{
I. Tzanakis ${ }^{1}$, M. Hadfield ${ }^{1}$, A. Georgoulas ${ }^{2}$ \& N. Kotsovinos ${ }^{2}$ \\ ${ }^{1}$ Sustainable Design Research Centre, School of Design, \\ Engineering and Computing, Bournemouth University, UK \\ ${ }^{2}$ Department of Civil Engineering, Democritus University of Thrace, \\ Greece
}

\begin{abstract}
Observations of cavitation damage within a lubricated expander system are studied experimentally. Typical experimental analysis is used to observe cavitation erosion features such as SEM, light-microscopy and lightinterferometer.

An experimental test-rig is used to study bubble characteristics within fluids to compare the theoretical analysis and practical in-service results from the expander system. Using an ultra-sonic methodology and high-speed camera techniques the bubbles are observed within the working fluids.

A 2D numerical simulation of the scroll was performed to explore the mechanism which generates scroll cavitation. It is found that the pressure is high enough to liquefy instantaneously part of the refrigerant close to the bottom boundary, creating conditions for the generation of cavitation bubbles within the liquefied refrigerant. This finding resolves the puzzle how the refrigerant which enters the scroll in gas phase produces cavitation.
\end{abstract}

Keywords: expander, scroll, refrigerant, lubricant, cavitation, erosion, bubbles.

\section{Introduction}

Scroll expander has been the main part of domestic appliances over the last few years (CHP systems, air-conditions, pumps etc). Many of these devices are facing cavitation wear problems because of their long operational period, their complicated geometry, their mechanical design and their high-speed working fluids. Interestingly, cavitation was identified by the author during the 
investigation of a specific scroll device in a previous study [1]. A small numbers of papers, published the last few years, are dealing with the operation of the scroll expander in an organic rankine cycle (ORC) system. The papers are mainly focused on the performance and the efficiency of the scroll expander rather than looking the tribological aspects of scroll's critical components during its operational period [2-7].

In this study a scroll expander which was under operational conditions for a thousand hours, as a main part of a small domestic combined heat and power (CHP) organic rankine system (ORC), is experienced damage in its main components which without any doubt identified as cavitation damage. The fluid environment consists of the two scroll fluids: the high molecular organic fluid (refrigerant) which mainly is in a gas form and drives the scroll and the synthetic oil (lubricant) which protects the parts of the scroll from excessive wear. The puzzle is which of the two fluids and by which mechanism the cavitation damage is produced. At first glance the pressure and temperature conditions inside the scroll expander are such that the lubricant liquid will not has chance to change phase from liquid to gas (to produce bubbles) and the refrigerant which is entering scroll as gas at high temperature (around $160^{\circ} \mathrm{C}$ ) is supposed to always remain in a gas state. Thus, the mean of generating cavitation by instantaneous transformation of phases from liquid to gas is not obvious. Not much of experimental data on the lubricant bubbles [8-10] and substantially fewer on the refrigerant bubbles $[11,12]$ relating to their velocity and impact attributes are available in literature.

The experimental study investigates the formation of the pits in their incubation period. The evaluation of the photographic results in correlation with the experimental results by the collapse impingement of the bubbles led to a more complete understanding of the cavitation erosion mechanisms. Using CFD methods the cavitation mechanism of the scroll expander was revealed. A 2D analysis of the scroll was performed. The aim was to investigate the behaviour of the refrigerant jet stream as it develops inside the first expansion chamber of the scroll. On the basis of numerical simulation results, it is advocated that the cavitation mechanism is related to the initial instantaneous high pressure at the lower scroll boundary induced by the refrigerant inflow gas jet. Instantaneously part of the refrigerant gas phase is transformed to liquid phase due to high pressure, which subsequently due to turbulent environment and high pressure fluctuations produces cavitation bubbles within the refrigerant in liquid phase.

\section{Experimental methodology}

Cavitation erosion tests were utilized in the present study to analyse and evaluate the performance of the cavitation bubbles generated within the two scroll working fluids. The experiments were performed according to the ASTM G3203 standard method. The ASTM method proposes a standard probe of $15.9 \mathrm{~mm}$ diameter to be generally used for cavitation tests. However, in this study a probe of $5 \mathrm{~mm}$ was deployed to produce cavitation bubbles. 
The steel plate of the scroll is machined into appropriate samples shape by a cutting machine. Then the steel samples were implemented on a Bakelite base. All the test samples were polished in a few microns accuracy and went through a surface analysis to ensure that they were free from any surface defects prior to testing. The chemical composition of the steel identified with the use of an optical emission spectrometer is shown in table 1 .

Table 1: Chemical analysis of the scroll's steel plate.

\begin{tabular}{|cccccccccc|}
\hline $\begin{array}{l}\text { Steel } \\
\text { Plate }\end{array}$ & $\% \mathrm{C}$ & $\% \mathrm{Mn}$ & $\% \mathrm{OSi}$ & $\% \mathrm{~S}$ & $\% \mathrm{P}$ & $\% \mathrm{Cu}$ & $\% \mathrm{Ni}$ & $\% \mathrm{Cr}$ & $\% \mathrm{Fe}$ \\
$\mathrm{Wt} \%$ & 0.96 & 0.53 & 0.27 & 0.003 & 0.021 & 0.01 & 0.02 & 0.18 & $\mathrm{REM}$ \\
\hline
\end{tabular}

The experimental apparatus is described in figure 1. Experiments were carried out by using an ultrasonic transducer at a frequency of $20 \mathrm{kHz}$. Ultrasonic horn has a plane surface oscillating in a simple harmonic motion. Peak to peak amplitude of the horn tip was adjusted to be at $50 \mu \mathrm{m}$. The sample was mounted on the bottom of a small transparent tank with a maximum capacity of $500 \mathrm{ml}$. The ultrasonic horn was submerged into the liquid environment of the tank in a distance of $0.5 \mathrm{~mm}$ from the chromium steel surface. Furthermore a high speed camera (Phantom v7.3) was installed at one of the sides of the tank. At the opposite side of the tank a high intensity fibre optic light source was implemented. Pictures were taken in random time steps during the cavitation process.

The liquids used for the tests were a synthetic lubricant and a high molecular organic refrigerant. All the liquids were tested in a room temperature of around $23 \pm 2^{\circ} \mathrm{C}$. The physical properties of the fluids used for the experiments are listed

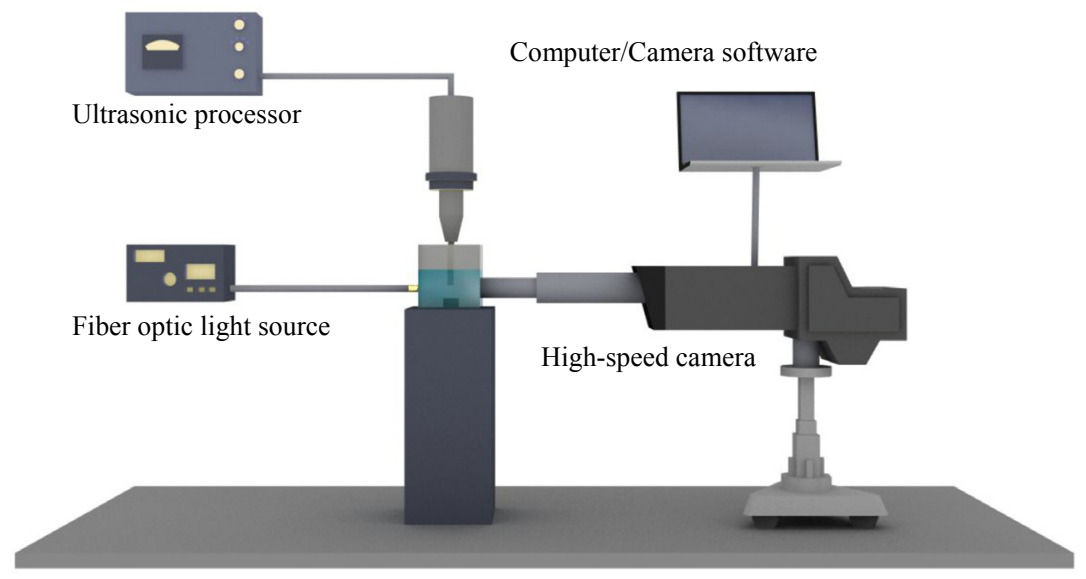

Figure 1: $\quad$ Schematic of experimental set up. 
Table 2: $\quad$ Physical properties of the experimental liquids at $25^{\circ} \mathrm{C} / 1 \mathrm{bar}$.

\begin{tabular}{|c|c|c|}
\hline Fluid & Refrigerant & Lubricant \\
\hline Ultrasonic Velocity $(\mathrm{m} / \mathrm{sec})$ & 990 & 1700 \\
\hline Liquid Density $\left(\mathrm{kg} / \mathrm{m}^{3}\right)$ & 625 & 995 \\
\hline Viscosity $(\mathrm{mPa} \cdot \mathrm{s})$ & 0.225 & 30 \\
\hline Surface Tension $(\mathrm{mN} / \mathrm{m})$ & $32-35$ & $49-54$ \\
\hline Vapour Pressure $(\mathrm{Pa})$ at $20^{0} \mathrm{C}$ & 53300 & 1300 \\
\hline
\end{tabular}

in table 2. The viscosity and surface tension for all the three fluids was measured using appropriate experimental devices.

Selected testing time was ten minutes, in order to prevent the temperature of the tested fluids from rising more than two to three degrees during the cavitation process. Furthermore, the amount of the incubation pits formed on the surface of steel samples was sufficient enough for analysis and interpretation. The temperature was controlled every minute with a laser digital thermometer.

\section{Surface investigation}

After the end of the process, the scroll expander of the CHP unit was disassembled and its high carbon steel plates were isolated and sent for surface investigation. This procedure was followed in order to analyse thoroughly the surface of the steel plate. Three different types of microscopes, an optical, an electronic (SEM) and an interferometer (ZYGO) microscope were deployed.

Interestingly, during the surface analysis of the scroll's steel plate, cavitation erosion was easily identified under the optical microscope. Many cavitation pit clusters were spotted across the high pressure regions of the steel plate, in the beginning of the expansion process. The cavities were mainly accumulated along the edges of the steel plate, creating a continuous layer of cavities of about $2 \mathrm{~mm}$ wide in both edges (figure 2). Surprisingly, on the low pressure regions of the steel plate, at the end of the expansion cycle, no trace of cavities was found.

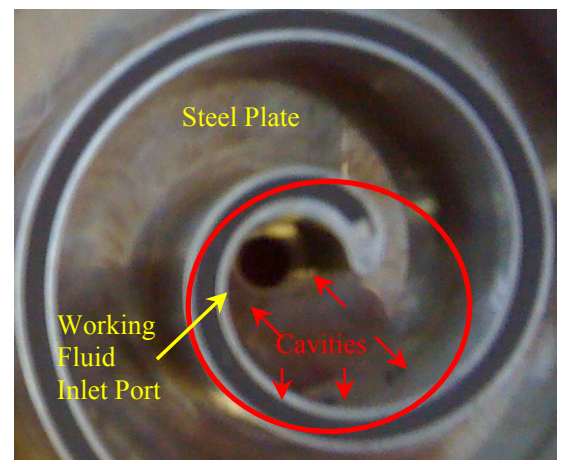

Figure 2: The high pressure area of the scroll expander. cavities formed across the edges (red arrows). 
The average width of the steel plate is around $12.5 \mathrm{~mm}$. However, in the areas where cavitation was revealed the width is even less, due to the geometrical profile of the scroll. Thus, the $2 \mathrm{~mm}$ width range of cavitation across the edges of the steel plate can be considered as a severe surface damage. The existence of cavities can rapidly increase the cavitation process creating deeper holes hence increasing the leakage and reducing the efficiency of the scroll.

In figure 3 a typical cavity found on the steel plate is analysed. The average depth of the individual cavities was estimated around 6-8 microns. In some cases the depth of the cavities was up to 10 microns. In addition, craters were formed due to a consistent and rigorous impact from the bubbles implosion inside a single cavity. Their enlargement into craters is a time and history dependent process.

a)

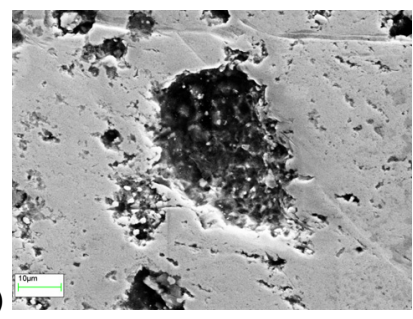

b)

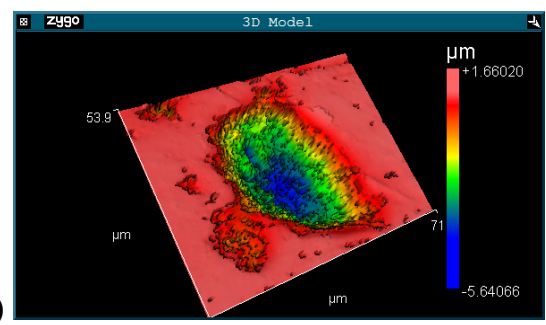

Figure 3: Cavities profile in detailed a) SEM microscope b) ZYGO interferometer.

\section{Test results}

The results produced were distinguished in two categories: the numerical and the experimental. The rational behind this was a coherent interpretation of the erosion mechanisms found on the steel plate surface. Initially, the numerical results produced with computational fluid mechanics (CFD) methods, offered by the commercial CFD software FLUENT, explain the generation of cavitation bubbles inside the scroll expander. The experimental results reveal the performance of the working fluids and their impact mechanisms on the actual steel plate of the scroll.

\subsection{Numerical results}

With the use of CFD methods, a finite volume numerical approach was applied in order to reveal the cavitation mechanism of the scroll expander. A 2D analysis of the scroll was performed. The aim was to investigate the behaviour of the jet stream as it develops inside the first expansion chamber of the scroll. It was believed that the cavitation mechanism was derived by the low pressure at the lower scroll boundary induced by the refrigerant. Thus, a drop of pressure by the entrainment was thought to be sufficient enough to make the lubricant volatile. Interestingly, only a small pressure drop was observed in the entrainment points while a tremendous increment of pressure was spotted in the scroll. 
The numerical model that was used in the simulations solves the Reynolds Averaged Navier Stokes equations (RANS). Closure of the turbulent stress terms is achieved with the application of the standard $\mathrm{k}-\varepsilon$ turbulence model. In more detail the equations solved are the continuity equation, the momentum equation, the energy equation and the transport equations for the turbulent kinetic energy (k) and its dissipation rate $(\varepsilon)$, respectively. At the solid boundaries of the geometry a wall boundary was applied, with enhanced wall treatment for the prediction of the turbulent quantities at the near-wall regions. A velocity-inlet boundary condition was used for the inlets and a pressure-outlet boundary condition for the outlets.

Two critical areas were chosen for the analysis. The first area is the moving wall (right side-outflow) at its minimum distance from the inlet port (inflow) in the very first stage of the expansion process. The second area is at the maximum operating distance of the moving wall (outflow) from the inlet port. In the first area a total number of 20493 quadrilateral cells were used, while the second area consists of a total number of 22050 quadrilateral cells. In both cases, grid clustering was used near the boundaries (figure 4).
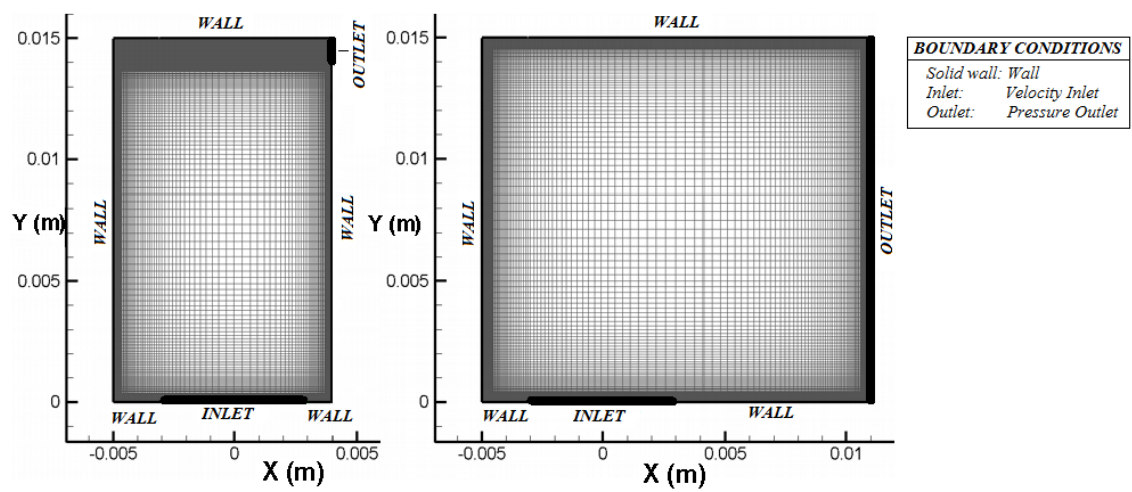

Figure 4: Geometry, computational mesh and boundary conditions of the first expansion pocket of the scroll expander.

At the early stage of the scroll operational mode there are no gaps created. Therefore, refrigerant is prevented from passing to the next pressurised chamber as it is introduced to the scroll. The refrigerant thrust pushes the scroll wall creating a gap. Thus, in the first case of the numerical analysis, a small gap was used for the equation of continuity to be solved. This was the worst scenario for generating high pressure fluctuations inside the scroll. In the second case, where the optimum scenario for avoiding cavitation due to pressure fluctuations is used, the gap is assumed to be across the whole side of the scroll wall, when the moving wall (outflow) is in its maximum possible operating distance from the inlet port. In both scenarios the shape and the operational conditions of the scroll expander were adjusted to the real world case, for representative results. Each pressure cycle according the operational mechanism of the scroll lasts around $17 \mathrm{msec}$. 


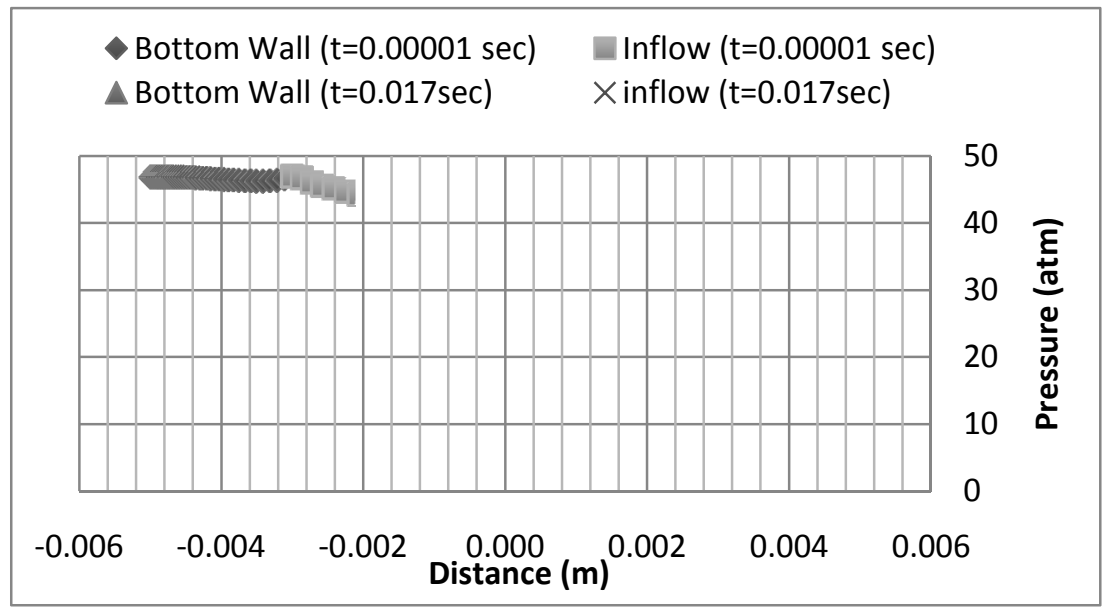

Figure 5: Pressure distribution along bottom boundary (initial position of moving part).

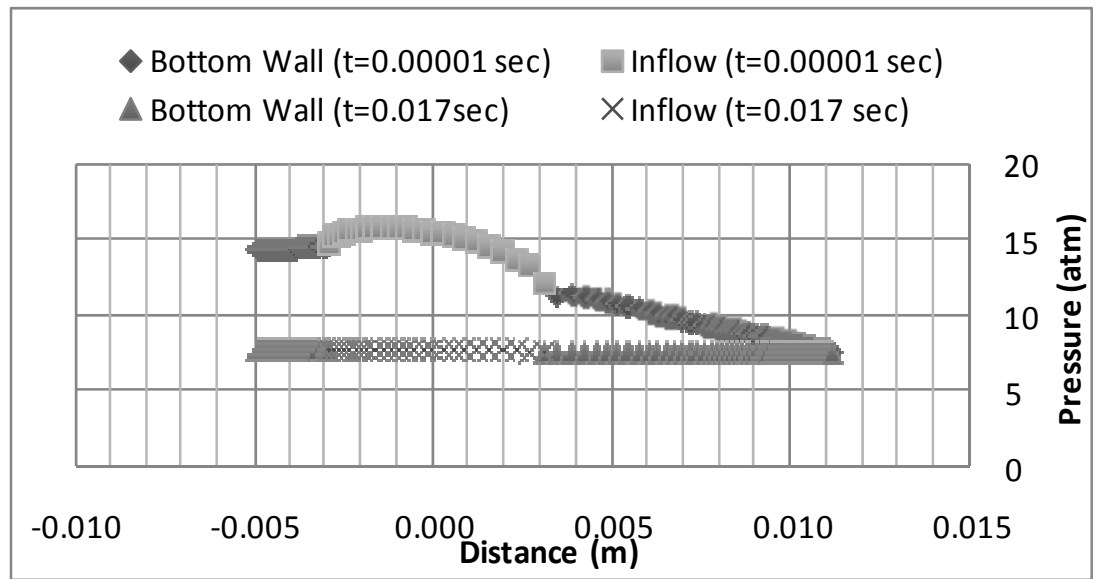

Figure 6: Pressure distribution along bottom boundary (final position of moving part).

The velocity of the inflow gas jet refrigerant is $31 \mathrm{~m} / \mathrm{sec}$. Subsequently in figures 5-6 the instantaneous pressure distribution for $\mathrm{t}=1 \mu \mathrm{sec}$ and $\mathrm{t}=17 \mathrm{msec}$ is presented, on the bottom plate where cavitation was found. From both figures 56 , for $\mathrm{t}=1 \mu \mathrm{sec}$, when the jet stream from the refrigerant is developed, there is a steep increment of the ambient pressure. In figure 5 the refrigerant pressure reaches for $\mathrm{t}=1 \mu \mathrm{sec}$ an extremely high level of around 45 bars across the bottom surface and for $\mathrm{t}>1 \mu \mathrm{sec}$ the pressure decreases and is stabilized at around 17 bars until the end of every pressure cycle. 
The same mechanism applies when the moving wall is at the maximum operating distance from the inlet port. As figure 6 shows, the ambient pressure across the bottom wall increases to 16 bars and then drops down to 8 bars. In both cases the pressure is high enough and the transformation of the lubricant from the liquid to gas phase is not possible. Therefore it seems that the lubricant is not responsible for the cavitation. However, in both cases the pressure is high enough to liquefy instantaneously part of the refrigerant close to the bottom boundary, creating conditions for the generation of cavitation bubble within the liquefied refrigerant. This finding resolves the puzzle how the cavitation erosion mechanism on the steel plate is produced.

\subsection{Experimental results}

The photographic results from the high speed camera revealed some interesting images regarding the performance of the lubricant and the refrigerant cavitation bubbles.

In the case of the lubricant, the bubbles were generated in low pressure areas and then collapsed in regions where the pressure was higher, creating severe damage on the surface of the material. Interestingly, a lubrication layer is generated during the cavitation process (figure 7). The lubrication layer can affect the implosion phenomenon of the bubbles. It can protect the material surface by acting as a cushion, reducing the impact from the bubbles implosion. Thus, despite the fact that the impact pressure from the lubricant bubbles is higher than in the refrigerant, the actual damage can be considerably less.

When refrigerant was used, bubbles had a remarkable behaviour while approaching the surface of the steel plate in figure 8 . They sustained a continuous oscillatory movement without imploding. A ceaselessly wobbling effect was also noted. Eventually the refrigerant bubbles obtained an amoeboid shape. Most of the refrigerant bubbles wobble instead of collapsing. Eventually no significant material loss or severe surface damage was caused. The surface tension of the refrigerant could possibly play an important role in this behaviour.

At the very early stages of the cavitation process, the impact energy released during cavity collapse on the surface is absorbed without any material deformation. Then, plastic deformation occurs which propagates into the grain boundary of the material displacing the grains' structure. Subsequently, within the ten minutes of the experimental process, the boundary grains are fractured, material loss is observed and cavitation pits are formed.

In figure 9 a typical destructive cavitation pit, produced in a lubricant and a refrigerant environment, is shown. The SEM image clearly illustrates the nature of the pit and its shape. The depth is close to the average typical depth produced by the impact of the lubricant and refrigerant bubbles respectively. The determinant between the two different types of pits is their depth level.

This is clearly depicted in figure 10 . The pits produced on the surface of the steel sample within the lubricant environment are on average 5 times deeper than the ones found on the refrigerant environment. In addition, the average depth of the larger pits produced by lubricant bubbles was found to be three times higher 


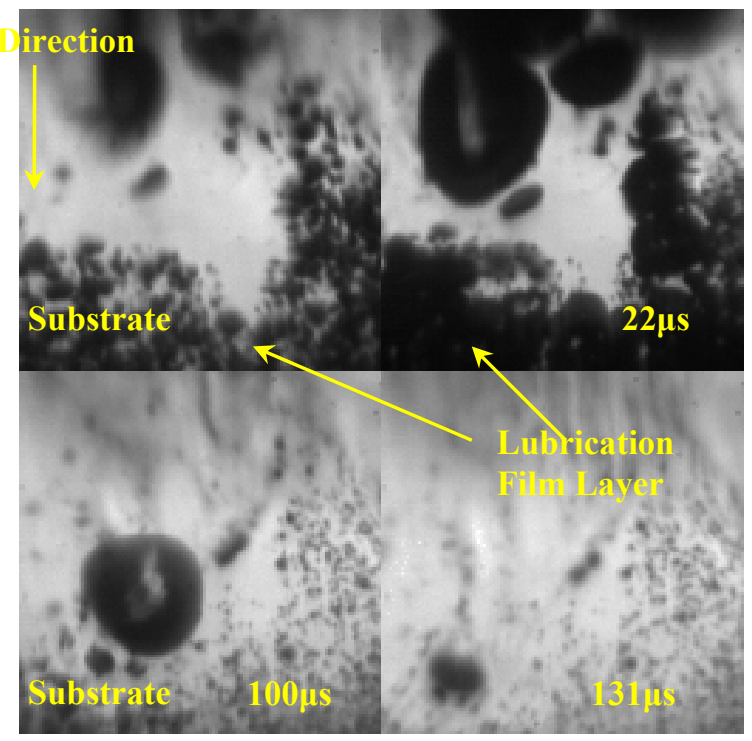

Figure 7: Collapse of an acoustic cavitation lubricant bubble near the solid boundary. A lubrication layer is formed. The frame size is $0.5 \times 0.5 \mathrm{~mm}[12]$.

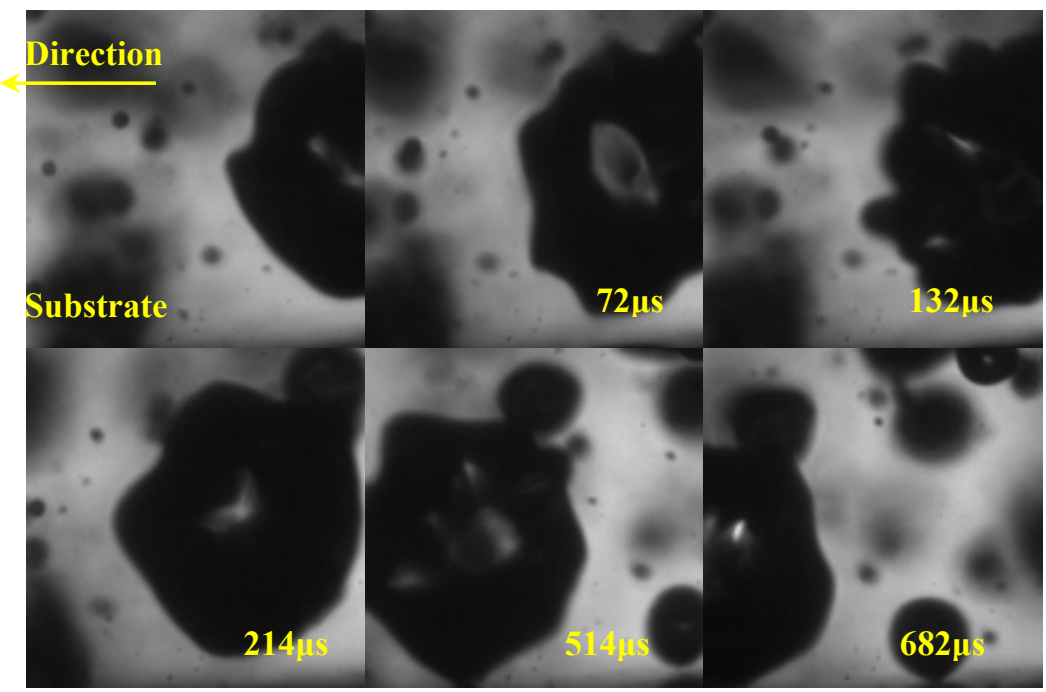

Figure 8: The ceaselessly wobbling movement of an acoustic cavitation refrigerant bubble near the solid boundary. The frame size is $0.5 \times 0.5 \mathrm{~mm}[12]$. 


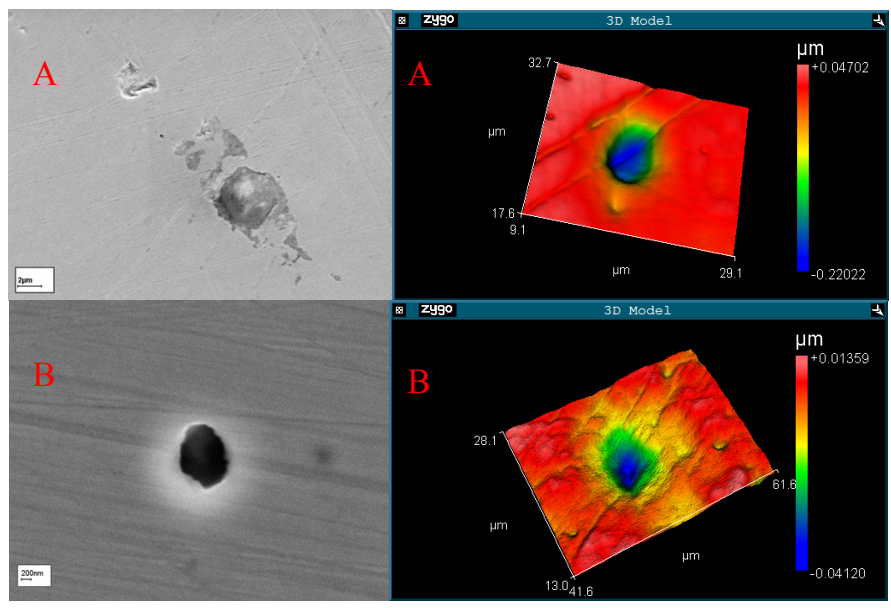

Figure 9: A typical erosion pit a) lubricant b) refrigerant.

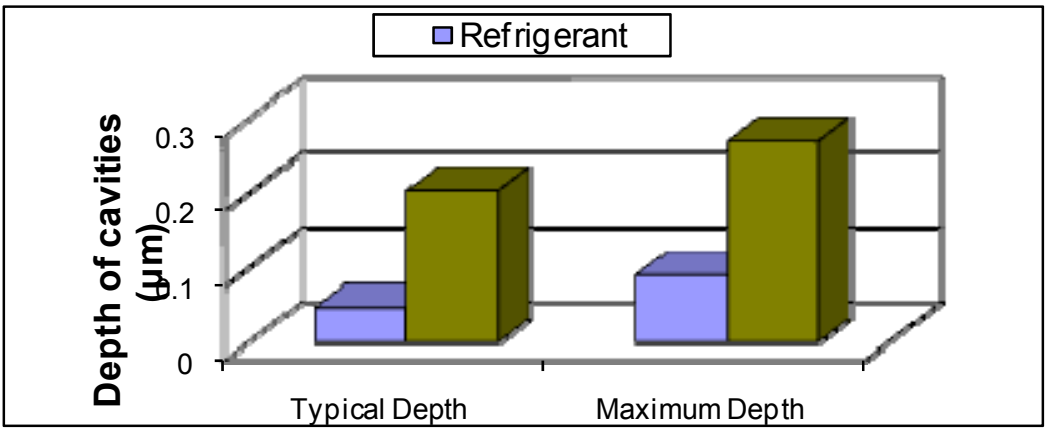

Figure 10: Depth of cavitation pits in different environments.

in comparison to the cavities generated by the implosion of the refrigerant bubbles.

\section{Discussion}

After the thorough surface investigation and the evaluation of the experimental and the numerical results, the cavitation mechanism responsible for damaging the surface of the scroll's steel plate is revealed. The initial consideration that cavitation derives from the lubricant, because its liquid phase was easier to generate bubbles, seems to weakening after the results produced from the computational fluid dynamic analysis. Interestingly, the refrigerant bubbles seem to be the main reason for the generation of cavities during the operational period of the scroll expander. The refrigerant bubbles are imploded near the boundary surfaces damaging the material. 
The refrigerant working fluid while entering the scroll and striking to the upper boundary produces a tremendous impact, resulting to a steep increase of the ambient pressure. This increment of pressure is sufficient enough to change its phase from gas to liquid. Moreover this ambient high pressure lasts only for 20 to $30 \mu \mathrm{sec}$. However, this time, having observed the generation of bubbles through the high speed camera, is adequate enough to produce cavities inside the bulk environment of the scroll.

Finally, the refrigerant molecules as they change phase from gas to liquid can accelerate the miscibility process with the lubricant inside the scroll. The vapour point of the lubricant can be significantly reduced according to the miscibility degree. At high miscibility degree, the lubricant becomes more volatile which in turn reduces the performance of the lubricant film thickness which results in increasing abrasion. Future work in this area will involve a systematic study of the refrigerant lubricant combination in order to define the physical properties under various temperature and pressure conditions.

\section{Conclusions}

It is reasonable to conclude from the results of this study that the responsible cavitation mechanism for the erosion of the scroll's steel plate was determined. Refrigerant is the fluid mean which generates cavitation bubbles due to elevated pressure fluctuations inside the high pressure gas pockets of the scroll expander as the analytical results revealed.

This finding resolves the puzzle how the refrigerant which enters the scroll in gas phase produces cavitation. This is really an exceptional situation. Usually we have cavitation in an environment where the fluid in motion is in liquid phase where at low pressure is transformed to gas producing cavitation bubbles. In our case, we have a situation that the fluid in motion is in gas phase, which under very high pressure conditions is locally liquefied, producing cavitation bubbles within the high pressure region. We are not familiar for a similar finding in the literature.

The experimental results from the ultrasonic transducer showed that the jet impact produced by the implosion of the refrigerant bubbles is less destructive compare to the lubricant bubbles. The depth of the pits is more significant in the lubricant environment compared to the refrigerant. Overall, refrigerant bubbles have a low impact pressure. However, over a prolonged period of time (1000 hours) the surface investigation results on the steel plate revealed the critical damage produced by the refrigerant bubbles.

\section{References}

[1] I. Tzanakis, M. Hadfield, Z. Khan. 2009. Durability of domestic scroll compressor systems. WIT Press 62 (229-240).

[2] M. Kane, D. Larrain, D. Favrat, Y. Allani. 2003. Small hybrid solar power systems. Energy 28 (1427-1443) 
[3] T. Yanagisawa, M. Fukuta, Y. Ogi, T. Hikichi. 2001. Performance of an oil-free scroll-type air expander. Proceedings of the IMechE Conference on Compressors and their Systems. (167-174).

[4] B. Aoun, D. Clodic. 2008. Theoretical and experimental study of an oil-free scroll type vapour expander. Proceedings of the Compressor Engineering Conference Purdue. (Paper 1188).

[5] S. Quoilin, V. Lemort, J. Lebrun. 2009. Experimental study and modelling of an Organike Rankine Cycle using scroll expander. Applied Energy.

[6] H.J. Kim, J.M. Ahn, S.O. Cho, K.R. Cho. 2008. Numerical simulation on scroll expander-compressor unit for CO2 trans-critical cycles. Applied Thermal Engineering 28 (1654-1661).

[7] V. Lemort, S. Quoilin, C. Cuevas, J. Lebrun. 2009. Testing and modelling a scroll expander integrated into an Organic Rankine Cycle. Applied Thermal Engineering 29 (3094-3102).

[8] E.A. Brujan, P.R Williams. 2006. Cavitation phenomena in non-Newtonian liquids. Chemical Engineering Research and Design 84 (293-299).

[9] P.R Williams, P.M. Williams, S.W.J. Brown. 1998. A study of liquid jets formed by bubble collapse under shock waves in elastic and Newtonian liquids. Journal of Non-Newtonian Fluid Mechanics. 76 (307-325).

[10] Y. Meged, C.H. Venner, W.E. ten Napel. 1995. Classification of lubricants according to cavitation criteria. Wear 186 (443-453).

[11] B. Schneider, A. Kosar, Y. Peles. 2007. Hydrodynamic cavitation and boiling in refrigerant (R-123) flow inside microchannels. International Journal of Heat and Mass Transfer 50 (2838-2854).

[12] I. Tzanakis, M. Hadfield. 2010. Observations of acoustically generated cavitation bubbles within typical fluids applied to a scroll expander lubrication system. Journal of Hydrodynamics. 